\title{
A comparison of complication rate between anterior and lateral approaches to the lumbar spine
}

\author{
Lumir Hrabalek ${ }^{a}$, Milan Adamus ${ }^{b}$, Adolf Grygac, Tomas Waneka, Peter Tucek ${ }^{a}$
}

\begin{abstract}
Aim. The aim of this study was to compare the complication rate of traditional minimally invasive anterior with the new minimally invasive lateral trans-psoatic retroperitoneal approaches to the intervertebral discs at levels T12-L5. Methods. A review of all cases of minimally invasive anterior (ALIF) and lateral (XLIF) intervertebral disc surgery at levels T12-L5, treated at the Department of Neurosurgery from January 1996 to September 2011. The ALIF group consisted of 120 and the XLIF group consisted of 88 patients. Preoperative diagnoses were: degenerative disc disease, failed back surgery syndrome, spondylolisthesis, retrolisthesis and posttraumatic disc injury. The surgical steps are described. All surgical intraoperative and postoperative complications directly related to the spinal surgery were prospectively documented. The outcome measure was rate of complications.

Results. In the ALIF group there were no major complications, only 35 minor intra- and postoperative complications in 32 patients (26.6\%). The main complication was lumbar post-sympathectomy syndrome in 19 patients (15.8\%). In the XLIF group there were 26 complications in 22 patients (25\%). One major intraoperative complication was partial and transient injury to the L5 nerve root (1.1\%). There were 25 minor postoperative complications in the XLIF group in 21 patients (23.9\%), mainly transient pain of the left groin or anterior thigh in 11 patients (12.5\%) or numbness in the same dermatomas in 9 patients (10.2\%). Statistically there was no difference between the ALIF and XLIF groups in complication rate.

Conclusion. Anterolateral and lateral retroperitoneal minimally invasive approaches to levels T12-L5 disc spaces are safe procedures with only minor complications and one exception. The rate of complications was similar in both groups. In the case of ALIF, the particular complication was post-sympathectomy syndrome. The main complication of XLIF was transient nerve root injury in one patient due to underestimation of the procedure in the outset. Intraoperative neuromonitoring during XLIF surgery is fully recommended.
\end{abstract}

Key words: lumbar spine, minimally invasive surgery, complications, interbody fusion

Received: February 11, 2012; Accepted with revision: July 19, 2012; Available online: September 5, 2012 http://dx.doi.org/10.5507/bp.2012.079

${ }^{a}$ Department of Neurosurgery, Faculty of Medicine and Dentistry, Palacky University Olomouc and University Hospital Olomouc, Czech Republic

${ }^{b}$ Department of Anesthesiology and Intensive Care Medicine, Faculty of Medicine and Dentistry, Palacky University Olomouc and University Hospital Olomouc

'Department of Surgery, Prostejov Hospital, Prostejov

Corresponding author: Lumir Hrabalek, e-mail: lumir.hrabalek@seznam.cz

\section{INTRODUCTION}

Lumbar interbody fusion using a supportive cage is a common surgical method for the treatment of degenerative lumbar spinal diseases and intervertebral disc injuries ${ }^{1,2}$. Improved surgical techniques for minimally invasive anterior approach to the lumbar spine (ALIF Anterior Lumbar Interbody Fusion) have led to renewed interest in this procedure ${ }^{3-5}$ and to the development of the new technique of lateral trans-psoatic minimally invasive access to the lumbar discs (XLIF - Extreme Lateral Interbody Fusion) (ref. ${ }^{6-8}$ ).

The aim of this study was to compare the rate of complications of the minimally invasive anterior (ALIF) with the minimally invasive lateral trans-psoatic (XLIF) retroperitoneal approaches to the intervertebral discs at levels T12-L5.

\section{MATERIAL AND METHODS}

We reviewed all cases of minimally invasive techniques (MISS - Minimally Invasive Spine Surgery) for anterior and lateral intervertebral disc surgery at levels T12-L5, treated at the Department of Neurosurgery from January 1996 to September 2011. The ALIF group consisted of 120 patients: 53 women and 67 men, average age 44 years, range 17-76 years. The XLIF group consisted of 88 patients: 50 women and 38 men, average age 51 years, range 17-74 years.

The patients suffered from low back pain and in most cases neither radiculopathy alone nor and conservative, traditional nonoperative management were effective for a minimum of 6 months. The diagnosis was established using history-taking and physical examination, radiography and magnetic resonance imaging (MRI) of the lumbosacral spine. 
One level ALIF surgery was indicated in 112 patients and two levels in 8 patients (altogether 128 disc surgeries). The preoperative diagnosis of the ALIF group was "Degenerative Disc Disease" (DDD) at 39 levels, "Failed Back Surgery Syndrome“ (FBSS) after previous disc herniation surgery without recurrence of disc herniation at 27, spondylolisthesis at 27 and posttraumatic disc injury at 35 levels. We operated 79 discs at levels L4-5, 19 discs at L3-4, 13 discs at L2-3, 14 discs at L1-2 and 3 discs at level T12-L1.

One level XLIF surgery was indicated in 84 patients and two levels in 4 patients (altogether 92 disc surgeries). The preoperative diagnosis of the XLIF group was DDD at 39, FBSS at 22, spondylolisthesis at 17, retrolisthesis at 5 and posttraumatic disc injury at 9 levels. We operated 3 discs at level L5-6, 57 discs at L4-5, 12 discs at L3-4, 11 discs at L2-3, 6 discs at L1-2 and 3 discs at level T12-L1.

Contraindicated were patients with severe osteoporosis, tumor, infection, fresh fracture of the spine, spondylolisthesis of grades III or IV and significant stenosis of the canal in both groups.

\section{Surgical steps \\ Anesthesia and positioning (ALIF, XLIF)}

Both types of surgery were performed retroperitoneally under balanced general anesthesia and adequate neuromuscular block for good surgical access; intravenous prophylactic application of antibiotics and low molecular weight heparin (LMWH) were obligatory. The patients were placed in a right lateral decubitus position on an adjustable surgical table and approaches were performed from the left side. The table was tilted to create a left convex bending of the lumbar spine. The skin was marked for skin incision under fluoroscopic control in order to achieve a parallel projection of the vertebral endplates of the level to be approached.

\section{Access to the lumbar spine, discectomy and implantation in ALIF}

Anterolaterally and obliquely from the left side a 5-7 $\mathrm{cm}$ skin and subcutaneous tissue incision was made. The retroperitoneal space was reached by a blunt, muscle splitting approach. Each muscle layer (external oblique, internal oblique, transverse abdominal muscle) was dissected in the direction of their fiber orientation. Care was taken to preserve the subcostal, iliohypogastric and ilioinguinal nerves between the layers of the internal and transverse abdominal muscles. Using blunt dissection with cottonoids and Langenbeck hooks the psoas muscle was then identified.

The anterolateral attachments of the psoas muscle were incised, sharply dissected and retracted laterally. The branches of the sympathetic chain were identified. Thus the lateral border as well as the anterior and lateral circumference of the anterior longitudinal ligament was exposed. The disc space level was then confirmed under fluoroscopic control. The retractor blades (Synframe, Synthes, USA) were then inserted anteriorly to retract retroperitoneum and great vessels (especially left iliac common vein) and posteriorly to retract psoas muscle in order to expose the anterolateral intervertebral circumference. The blades were fixed to the ring of Synframe and fastened to the table.

Starting from the anatomical midline of the anterior longitudinal ligament, the annulus fibrosus was incised in its anterolateral circumference. The sympathetic chain was separated and retracted anteriorly or posteriorly. The disc was emptied with rongeurs and the endplates were curretted and carefully removed with chisels. The subchondral bone was smoothed with a high-speed drill. We used titanium spacers (Syncage, Synthes, USA, or Harms mesh, Johnson and Johnson, USA) or PEEK (poly-etherether-keton) spacers (Visios, Synthes, USA, or Antelys, Scient ' $x$, France) for ALIF. Cancellous bone from the iliac crest as well as from the removed parts of the vertebral bodies and artificial bone (ChronOs, Synthes, USA) were impacted into the spacer as well as around the cage for fusion. Cancellous bone chips were harvested from the anterior iliac crest usually through the same skin incision. We used Active-L (B-Braun, Germany) implant for TDR (Total Disc Replacement). The retractor was removed and the suction drain was inserted retroperitoneally. The muscle layers, subcutaneous tissue and skin were sutured.

\section{Access to the lumbar spine, discectomy and implantation in XLIF}

The skin and subcutaneous tissue were incised to a length $5-7 \mathrm{~cm}$ laterally and obliquely from the left side. The abdominal musculature was divided with blunt dissection by carefully splitting each muscular layer (external oblique, internal oblique, transverse abdominal muscle) and spare nerve structures (subcostal, iliohypogastric and ilioinguinal nerves). After accessing the retroperitoneal space, the peritoneum was gently swept anteriorly and the dissection was carried bluntly down to the psoas muscle.

The muscle fibers of the psoas were separated gently at the level of the disc under visual kontrol. The aim was avoid lateral femoral cutaneous nerve (LFCN), genitofemoral nerve and nerve roots. When the psoas muscle was divided to the anterior and posterior part, exposure of the lumbar spine was sufficient. The surgeon inserted a $\mathrm{K}$ - wire to the disc, over the K-wire dilators and special retractor (Oracle) with firm fixation to the operating table. A tubular retractor was fixed over the anterior and middle third of intervertebral disc space. Then the surgeon traced the course of the lumbar nerve roots with a stimulation electrode using triggered electromyography (tEMG) for intraoperative neuromonitoring (IOM). If the nerve roots were far from the blades of the retractor and intervertebral disc, a safe discectomy was possible via direct vision.

Discectomy and endplate preparation using special instruments, then interbody distraction and PEEK implant placement (Oracle, Synthes, USA) were performed. Artificial bone (ChronOs strip, Synthes, USA) filled by the patient's blood and bone marrow were impacted into the spacer and the interbody fusion was completed by packing the interspace. The incision was closed using standard technice and the use of suction drain was unnecessary. 


\section{Postoperative care and follow-up (ALIF, XLIF)}

Typically, patients were mobilized early and allowed to walk within $48 \mathrm{~h}$ of surgery. In cases of interbody fusion, bending, twisting, heavy lifting, and heavy exercise were restricted and lumbar orthosis was recommended to help reduce motion while fusion occurred. Patient with TDR had no restrictions. The surgeon examined the patients and radiographs were obtained at follow-up examinations of 6 weeks, 6, 12 and 24 months.

The decision to include concomitant anterior plating or posterior spinal fusion and instrumentation was individualized to the patient and pathology.

All surgical intraoperative and postoperative complications directly related to the spinal surgery were prospectively documented. In all patients with signs and symptoms of lumbar sympathectomy, thermography as a sensitive test was done minimally 2 months after surgery. Complications related to the implant healing were not included in the study. The minimum follow-up required for inclusion was 6 months. The Fisher exact probability test was used to compare the rate of complications at the $P=0.05$ level.

\section{RESULTS}

There were no major complications for the ALIF group. We found 35 minor intra- and postoperative complications in the ALIF group in 32 patients (26.6\%). Intraoperative complications were small peritoneal opening without visceral injury in three patients $(2.5 \%)$, pleural opening at level T12-L1 in one patient (0.8\%), injury to the iliolumbal vein without excessive bleeding and with subsequent ligation in one patient $(0.8 \%)$. The main postoperative complication was lumbar post-sympathectomy syndrome in 19 patients (15.8\%). At level L4/5 in 15 cases ( $19 \%$ of all ALIF surgeries at this level), at levels L $3 / 4$ and L2/3 each in two cases. Other minor postoperative complications were numbness of the left anterolateral thigh or groin in 6 patients $(5 \%)$, transient pain of the left groin in 4 patients $(3.3 \%)$ and seroma of the wound from the donor side in one patient $(0.8 \%)$.

In the XLIF group there were 26 complications in 22 patients (25\%). One major intraoperative complication was partial and transient injury to the L5 nerve root during implant insertion at level L4-5 (1.1\%). IOM was not yet used in this first case. There were 25 minor postoperative complications in the XLIF group in 21 patients (23.9\%), mainly transient pain of left groin or anterior thigh, in 11 patients $(12.5 \%)$ and numbness of left anterolateral thigh or groin, in 9 patients $(10.2 \%)$. Other minor postoperative complications were lumbar post-sympathectomy syndrome in 4 patients (4.5\%), including 3 cases at level L4/5 (5\% of all XLIF surgeries at this level) and one case at level L5/6 (33\% of all surgeries at this level).

In neither group were there any serious complications such as death, excessive intra- or postoperative bleeding, thrombembolism, infection, visceral injury including in- jury to the ureter or kidney, ileus, retrograde ejaculation or meralgia paresthetica.

Statistically (Fisher's Exact Test) there was no difference between ALIF and XLIF groups in rate of complications.

"Adjacent segment disease“ (always disc herniation) was found in three cases 4 or 5 years after ALIF (2.5\%). We have not yet operand on any patient with „adjacent segment disease" after XLIF. At minimally 6 months follow-up we found on X-ray no hardware failure, as implant displacement in either group. 198 patients were satisfied with the surgery and reported improvement, 10 patients were not improved or were worsened (radiological and clinical results were similar in both groups).

\section{DISCUSSION}

An anterior approach to the lumbar spine and ALIF was first performed and described in the 1930s (ref. ${ }^{9-11}$ ). Since then ALIF has become a popular procedure for degenerative disc disease, FBSS and spondylolisthesis . The literature on surgery-related complications of ALIF has largely focused on issues related to graft, instrumentation, and fusion outcomes. In few reports have authors analyzed the general surgery-related complications in any great detail ${ }^{12,13}$.

Faciszewski et al. reported that $58 \%$ of all anterior thoracolumbar procedures were free of complications during hospitalization or follow-up. They found that the risk of a complications increased for patients who were female, patients over age 60 years, and those with comorbidities $^{12}$. On the other hand, Tiusanen et al. reported in 1996 a detailed clinical outcome study in a series of 83 patients and the general-surgery related complication rate for ALIF was $80 \%$. Complications included unintentional sympathectomy (43\%), retrograde ejaculation (in $24 \%$ of males), venous injury (18\%), deep vein thrombosis (5\%), and infection (3\%) (ref. $\left.{ }^{14}\right)$. Most complications of ALIF associated with approach, were vessel injuries, post-sympathectomy syndrome and retrograde ejaculation.

There was no injury to great vessels in our study as we used a gentle technique of dissection and retractors to avoid to injury of surrounding structures. Thus, the vascular injury frequently described in the literature is not a real risk for an experienced surgical team. The reported incidence of venous injury ranges from $0 \%$ to $18 \%\left(\right.$ ref., $\left.^{4,14-17}\right)$. Venous laceration is the most common type of vascular injury due to vessel retraction. Other situations related to venous injury include discectomy and placement of interbody graft. The most frequently injured vessels are the left common iliac vein, inferior vena cava, and the iliolumbar vein. Occasionally, a deep vein thrombosis develops at the site of venous laceration. Most venous injuries in ALIF involve the L4-5 disc space. Baker et al. reported a 15\% incidence of vascular complications following ALIF in 85 patients. They found an increased incidence of venous injury when the retroperitoneum was approached via a small paramedian incision compared with a longer flank 
incision ${ }^{15}$. Similarly, injury to the common iliac vein or inferior vena cava was reported by Westfall et al. to occur in $15.6 \%$ of cases, and a much higher rate of great vessel injury was reported with rectus incision (18.4\%) versus flank incision $\left(7.7 \%\right.$ ) (ref. $\left.{ }^{17}\right)$. The identification and ligation of the iliolumbar vein in the early phase of the exposure has been recommended to prevent inadvertent avulsion of the vein off the vena cava later in the procedure $^{18}$. A cadaveric study showed that there are variations in the anatomy of the iliolumbar vein, and the presence of multiple iliolumbar veins may increase the chance of inadvertent venous injury ${ }^{19}$. XLIF - the more lateral trajectory compared to that of ALIF does not require retraction of the great vessels for exposure.

Among vascular complications of ALIF, the incidence of arterial injury is reported to be from $0 \%$ to $5.2 \%$ and the most common artery is the left common iliac artery $y^{4,20-22}$. Brau et al. cautions that thrombosis with occlusion of the common iliac artery after ALIF can be a rare complication which has been noted in $0.45 \%$ of cases and emphasized that surgeons must check the pulse of the dorsalis pedis artery peri- and postoperatively ${ }^{20}$. Kulkarni et al. reported in 8 patients $(5.2 \%)$ who had undergone mini-open ALIF, arterial complications. All cases involved surgery at the L4-5 level ${ }^{22}$. Mayer, the pioneer spine surgeon for mini-open ALIF, attributed the high complication rate of the study by Kulkarni et al. to the technical error of inappropriate vascular retraction ${ }^{4}$.

Lymphocoele is a rare complication but should be suspected if fluid collects postoperatively following anterior lumbar spine procedures. Lymph vessel anatomy should be borne in mind. Lymph vessels and nodes should be clearly identified and avoided. They are located lateral to the left common iliac artery and can be injured during dissection of the left ascending lumbar vein while exposing the L4-5 or L3-4 disc space ${ }^{23}$.

The main postoperative complication in our group of ALIF patients was post-sympathectomy syndrome (16\%) and the reason was usually the necessity to sacrifice the lumbar sympathetic chain to obtain wide access to the discs. Sometimes injury to the lumbar sympathetic chain located on the anterolateral aspect of the vertebral body was recognized in the past. Characteristically, the patient complaints of a cold limb or foot on the side contralateral to the approach. This is because the affected side is warmer due to loss of sympathetic vasoconstriction. The reported incidence of sympathetic dysfunction after ALIF is from $9 \%$ to $43 \%$ (ref. ${ }^{13,14,24}$ ). The danger of injury to the superior hypogastric plexus of the sympathetic system which in men results in retrograde ejaculation, is usually from $0 \%$ to $10 \%$ when operating using the retroperitoneal approach $^{2,13,24}$.

Nerve root injury or ureteral injury in the ALIF procedure are exceptional. Faciszewski et al. reported lumbar plexus injury in one patient resulting from excessive surgical dissection posterior and lateral to the psoas muscle. Ureteral laceration occured in a patient who had a previous anterior spinal surgery ${ }^{12}$. Stulík et al. found that injury to the ureter was associated with vascular trauma in one patient ${ }^{25}$.
The first trans-psoatic endoscopic approach was desribed by Pimenta in 2001 (ref. $^{8}$ ) and published by Bergey et al. in 2004 (ref. $^{6}$ ). XLIF using retractors was first described by Ozgur et al. in 2006 ( ref. $^{7}$ ). Thus, XLIF is a new technique and can be used to gain access to the lumbar spine via a lateral approach that passes through the retroperitoneal fat and psoas major muscle. In this way, the potential complications with an anterior approach to the lumbar spine (for ALIF) can be avoided. The great vessels, peritoneal contents and sympathetic chain are not usually encountered and the lateral approach should be safer. Rodgers et al. reported on a series of 600 XLIF patients. There were no wound infections, no vascular injuries and no intraoperative visceral injuries ${ }^{26}$.

However, there are limitations associated with XLIF techniques. The anatomic location of the ribs and of the iliac wing can limit the exposure and location of the nerves of the lumbar plexus are at risk. Motor deficits are the area of greatest concern to spinal surgeons and have been discussed frequently in relation to the lateral approach to the spine. Bergey et al. found using the lateral trans-psoas approach without IOM postooperative paresthesia rates as high as $30 \%$ (ref. $^{6}$ ). Prevention of nerve root injury is achieved through the use of IOM. The role of IOM of nerve roots during the placement of pedicle screws is well known, but in minimally invasive interbody fusion approaches has been less well studied or defined. In general, tEMG thresholds for response below $5 \mathrm{~mA}$ indicate direct contact, between 5 and $10 \mathrm{~mA}$ indicate close proximity (little, but some, soft tissue between the instrumentation and the nerve), and more than $11 \mathrm{~mA}$ indicate further distance from intrapsoas nerves ${ }^{27,28}$. Despite IOM, Knight et al. reported two cases $(3.4 \%)$ of permanent motor deficits thought to be due to injury to the L4 root $^{28}$. Rodgers et al. found motor deficits in four patients $(0.7 \%)$ treated at L4-5. Premedication of the patients undergoing surgery at L4 to L5 levels with dexamethasone $10 \mathrm{mg}$ IV before surgery has significantly reduced the incidence of these transient motor deficits ${ }^{26}$. Since our case of L5 nerve root injury we began strict use of IOM and we have had no more nerve roots complications.

The safe zone for performing discectomy and XLIF is located between nerve roots which run along the posterior part of the vertebral bodies (VB) and the sympathetic chain, which runs along the anterior third of VB. Uribe at al. divided the area between the anterior and posterior edges of the VB into 4 equal zones (fourths), ventroposteriorly marked as I.-IV. At level L1-2 all nerve roots are in zone IV. At level L2-3 and L3-4 is the genitofemoral nerve in zone I. and nerve rooots in zone IV. At level L4-5 are nerve roots (and femoral nerve) in zone III. and IV. and genitofemoral nerve in zone I. This means that, for nerve roots at levels L1-3 safe working zones are I. - III. and at level L4-5 the safe working zone are on the border of zones II. - III. ( ref. $^{29}$ ).

An area of concern in lateral-access spinal surgery is postoperative groin and thigh pain, the main complication in our XLIF group (12.5\%). Pain and hip flexor weakness are nearly universal due to direct trauma to the psoas muscle with swelling but this is always transient. On the 
other hand, hypesthesia in this area of the groin and thigh is likely the result of injury of to the iliohypogastric or ilioinguinal nerve during preparation through the abdominal wall. The hypesthesia usually spontaneously disappears. Dakwar et al. reported transient postoperative anterior thigh numbness, ipsilateral to the side of approach in $12 \%$ of patients ${ }^{30}$, Oliveira et al. in $14.3 \%$ of patients ${ }^{31}$ and in our study in $10 \%$. Knight et al. descibed 6 cases of meralgia paresthetica as a result of LFCN injury. In general, LFCN lies anterior to the psoas muscle within the retroperitoneal space and passes under inguinal ligament to the thigh. IOM using tEMG cannot detect sensory nerves and their changes (iliohypogastric, ilioinguinal nerves, LFCN) and attempts at using dermatomal somatosensory-evoked potentials have not been effective owing to the sensitivity of these signals to anesthetic agents ${ }^{28}$.

In contrast to the ALIF studies, retrograde ejaculation in men has not been yet reported after XLIF, including our study group.

Reported retroperitoneal minimally invasive approaches to levels of T12-L5 disc spaces in this study are safe procedures with only minor complications (except one major). The rate of complications in the groups was similar $(26.6 \%$ and $25 \%)$. The particular risk of ALIF is sympathectomy as, we need to obtain wide access to the disc anterolaterally and as a result it is often not possible to avoid surgical trauma to the lumbar sympathetic chain. Generally, the main advantage of XLIF over ALIF is reduced probability of vascular complications and sympathectomy, that is, the post-sympathectomy syndrome and retrograde ejaculation. The risk of XLIF is usually nerve root injury, but we are potentially able to prevent this using IOM and dexamethasone.

\section{CONCLUSION}

Anterolateral and lateral retroperitoneal minimally invasive approaches to levels of T12-L5 disc spaces are safe procedures with only minor complications (with one exception).

The rate of complications was similar in the two groups, $26.6 \%$ in ALIF group and 25\% in XLIF group of treated patients.

The main complication of ALIF was post-sympathectomy syndrome.

The main complication of XLIF was transient nerve root injury in one patient due to underestimation of this procedure in the beginning. IOM during XLIF surgery is fully recommended.

\section{REFERENCES}

1. Crock HV. Anterior lumbar interbody fusion: indications for its use and notes on surgical technique. Clin Orthop 1982;165:157-63.

2. Zdeblick TA, David SM. A prospective comparison of surgical approach for anterior L4-L5 fusion. Spine 2000;25:2682-7.

3. Mayer HM. A new microsurgical technique for minimally invasive anterior lumbar interbody fusion. Spine 1997;22:691-700.
4. Mayer HM, Wiechert K. Microsurgical anterior approaches to the lumbar spine for interbody fusion and total disc replacement. Neurosurgery 2002;51(Suppl 5):S159-65.

5. Mehren C, Mayer HM, Siepe C, Grochulla F, Korge A. The minimally invasive anterolateral approach to L2-5. Oper Orthop Traumatol 2010;22:221-8.

6. Bergey DL, Villavicencio AT, Goldstein T, Regan JJ. Endoscopic lateral transpsoas approach to the lumbar spine.Spine 2004;29:1681-8.

7. Ozgur BM, Aryan HE, Pimenta L, Tailor WR. Extreme lateral interbody fusion (XLIF): a surgical technique for anterior lumbar interbody fusion.Spine J 2006;6:435-43.

8. Pimenta L. Lateral endoscopic transpsoas retroperitoneal approach for lumbar spine surgery. Paper presentation at the VIII. Brazilian Spine Society Meeting 2001. Belo Horizonte, Minas Gerais, Brazil.

9. Ito H, Tsuchiya J, Asami G. A new radical operation for Pott's disease. J Bone Joint Surg 1934;16:499-515.

10. Mercer W. Spondylolisthesis: with a description of a new method of operative treatment and notes of ten cases. Edinburgh Med J 1936;43:545-72.

11. Speed K. Spondylolisthesis. Treatment by anterior bone graft. Arch Surg 1938;37:175-89.

12. Faciszewski T, Winter RB, Lonstein JE, Denis F, Johnson L. The surgical and medical perioperative complications of anterior spinal fusion surgery in the thoracic and lumbar spine in adults. Spine 1995;20:1592-9.

13. Rajaraman V, Vingan R, Roth P, Heary RF, Conklin L, Jacobs GB. Visceral and vascular complications resulting from anterior lumbar interbody fusion. J Neurosurg (Spine 1) 1999;91:60-4.

14. Tiusanen H, Seitsalo S, Osterman K, Soini J. Anterior interbody lumbar fusion in severe low back pain. Clin Orthop Relat Res 1996;324:153-63.

15. Baker JK, Reardon PR, Reardon MJ, Heggeness MH. Vascular injury in anterior lumbar surgery. Spine 1993;18:2227-30.

16. Brau SA: Mini-open approach to the spine for anterior lumbar interbody fusion: description of the procedure, results and complications. Spine J 2002;2:216-33.

17. Westfall SH, Akbarnia BA, Merenda JT, Naunheim KS, Connors RH, Kaminski DL, Weber TR. Exposure of the anterior spine. Technique, complications, and results in 85 patients. Am J Surg 1987;154:700-4.

18. Inamasu J, Guiot BH. Vascular injury and complication in neurosurgical spine surgery. Acta Neurochir 2006;148:375-87.

19. Jasani $V$, Jaffray $D$. The anatomy of the iliolumbar vein. A cadaver study. J Bone Joint Surg Br 2002;84:1046-9.

20. Brau SA, Delamarter RB, Schiffman ML, Williams LA, Watkins RG. Left iliac artery thrombosis during anterior lumbar Sumery. Ann Vasc Surg 2004;18:48-51.

21. Kim JS, Choi KC, Jung B, Lee SH. Thrombosis of left common iliac artery following anterior lumbar interbody fusion: case report and review of literatures. J Korean Neurosurg Soc 2009;45:249-52.

22. Kulkarni SS, Lowery GL, Ross RE, Ravi Sankar K, Lykomitros V. Arterial complications following anterior lumbar interbody fusion: report of eight cases. Eur Spine J 2003;12:48-54.

23. Schizas C, Foko'o N, Matter M, Romy S, Munting E. Lymphocoele: a rare and little known complication of anterior lumbar surgery. Eur Spine J 2009;18(Suppl 2):S228-31.

24. Saraph V, Lerch C, Walochnik N, Bach CM, Krismer M, Wimmer C. Comparison of conventional versus minimally invasive extraperitoneal approach for anterior lumbar interbody fusion. Eur Spine J 2004; 13:425-31.

25. Štulík J, Vyskočil T, Bodlák P, Šebesta P, Kryl J, Vojáček J, Pafko P. Poranění velkých cév při předním přístupu k hrudní a bederní páteři. Acta Chir Orthop Traumatol Čech 2006;73:92-8.

26. Rodgers WB, Gerber EJ, Patterson J. Intraoperative and early postoperative complications in extreme lateral interbody fusion.Spine 2010;36:26-33.

27. Uribe JS, Vale FL, Dakwar E. Electromyographic monitoring and its anatomical implications in minimally invasive spine surgery. Spine 2010;35:S368-74.

28. Knight RQ, Schwaegler $P$, Hanscom D, Roh J.Direct lateral lumbar interbody fusion for degenerative conditions: early complication profile.J Spinal Disord Tech 2009;22:34-7.

29. Uribe JS, Arredondo N, Dakwar E, Vale FL. Defining the safe working zones using the minimally invasive lateral retroperitoneal transpsoas approach: an anatomical study. J Neurosurg Spine 2010;13:260-6. 
30. Dakwar E, Cardona RF, Smith DA, Uribe JS. Early outcomes and safety of the minimally invasive, lateral retroperitoneal transpsoas approach for adult degenerative scoliosis. Neurosurg Focus 2010;28:E8.
31. Oliveira L, Marchi L, Coutinho E, Pimenta L. A radiographic assessment of the ability of the extreme lateral interbody fusion procedure to indirectly decompress the neural elements.Spine 2010;35:S331-7. 